\title{
Proper motion and kinematics of the ansae in NGC7009
}

\author{
Rodrigo Fernández M.1 ${ }^{1}$ \\ Pontificia Universidad Católica de Chile, Casilla 306, Santiago 22, Chile \\ Hektor Monteiro, Hugo E. Schwarz \\ Cerro Tololo Inter-American Observatory, NOAO, Casilla 603, La Serena, Chile. ${ }^{2}$
}

\begin{abstract}
We have measured the proper motion (PM) and kinematics of the ansae in NGC 7009 using high dispersion echelle spectra and archive narrow band HST images. Assuming that the ansae are moving at equal and opposite velocities from the central star we obtain a system radial velocity of $-53 \pm 2 \mathrm{~km} . \mathrm{s}^{-1}$, the eastern ansa approaching and the western ansa receding at $v_{r}=5.3 \pm 1 \mathrm{~km} . \mathrm{s}^{-1}$ with respect to this value. The PM of the eastern ansa is $28 \pm 8 \mathrm{mas}_{\mathrm{yr}}{ }^{-1}$, which with our weighted distance to NGC 7009 of $0.86 \pm 0.34 \mathrm{kpc}$ gives $V_{\exp }=112 \pm 32$ $\mathrm{km} . \mathrm{s}^{-1}$. The electron temperature and density in both ansae were determined to be $T_{e} \sim 9000 \pm 400 \mathrm{~K}$ and $n_{e} \sim 2300 \pm 400 \mathrm{~cm}^{-3}$. The dynamic age of the ansae is $\sim 925 \pm 260 \mathrm{yrs}$. and the implied PM of the central star is $\mu_{C S}=1 \pm 0.5$ mas. $\mathrm{yr}^{-1}$. This is in qualitative but not quantitative agreement with previous work.
\end{abstract}

Subject headings: astrometry — ISM: kinematics and dynamics — planetary nebulae: individual (NGC7009)

\section{Introduction}

Most low and intermediate mass stars become White Dwarfs after a brief phase as Planetary Nebulae (PNe) caused by heavy mass loss on the AGB (Iben \& Renzini 1983).

\footnotetext{
${ }^{1}$ CTIO REU-PIA Program student

${ }^{2}$ Cerro Tololo Inter-American Observatory, National Optical Astronomy Observatory, operated by the Association of Universities for Research in Astronomy, Inc., under a cooperative agreement with the National Science Foundation.
} 
Symmetrical and mildly asymmetrical PNe are explained by versions of the interacting wind model (Kwok et al. 1978) but the mechanisms causing the strong asymmetries observed in the majority of PNe are still unexplained. That both binaries and magnetic fields probably play a role is now generally accepted. For an overview of formation mechanisms, models, and asymmetries see Kastner et al. (2000).

The wealth of different PNe shapes is shown in Balick (1987) and Schwarz et al. (1992), and features have been described in detail by many authors: e.g. point symmetry (Schwarz 1993), bipolarity (e.g. Corradi \& Schwarz 1995), multipolarity (Sahai \& Trauger 1998), BRETS (López 1997) and Fast, Low Ionization Emission Regions or FLIERS (Balick et al. 1994) are all used to indicate the various observed morphologies.

NGC7009 has long been known to have a complex morphology. Aller (1941) described the outer features as ansae (handles in Latin), and work by Reay \& Atherton (1985), and Balick et al. (1987) show that these ansae are expanding near the plane of the sky at high velocities $\left(\sim 10^{2} \mathrm{~km} / \mathrm{s}\right)$ relative to the nucleus.

Liller (1965) used photographic plate material to analyze the expansion of NGC7009,

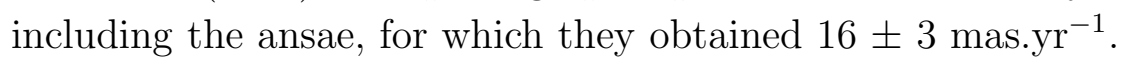

In this paper we present the kinematics and proper motion of the ansae using observational material from the HST and CTIO $4 \mathrm{~m}$ echelle spectrograph, and show that the previous results are qualitatively but not quantitatively correct.

\section{Observations and data reduction}

We extracted two sets of archive HST images with the "On the fly" option. The WFPC2 sets were taken on 1996.04.28 (Balick et al. 1998) and on 2001.05.11 (Palen et al. 2002) in an $\mathrm{H} \alpha /[\mathrm{NII}]$ filter.

The 2001 images are shifted 20" east from the 1996 images. As the latter covered the entire nebula, in the former the west ansa disappears from the field of view. The two images showed in Figure 1 are an average (for cosmic ray rejection) of each set.

We took CTIO 4m echelle spectra on 2002.07.29 with a spectral range between 410$720 \mathrm{~nm}$, and a slit of $1.2^{\prime \prime} \times 6.6^{\prime \prime}$. The mean seeing was $1.4^{\prime \prime}$. Flux calibration to $10 \%$ was done with $58 \mathrm{Aql}$ (HR 7596, Hamuy et al. 1992). The spectra were reduced in the usual way, using IRAF, and resulted in an rms wavelength error of $0.00037 \mathrm{~nm}$, with residuals of $\leq 0.00075 \mathrm{~nm}$. Velocity resolution was $3.7 \mathrm{~km} \cdot \mathrm{s}^{-1} \cdot \mathrm{pix}^{-1}$ or $10.2 \mathrm{~km} . \mathrm{s}^{-1}$ for our slit width at $650 \mathrm{~nm}$. 

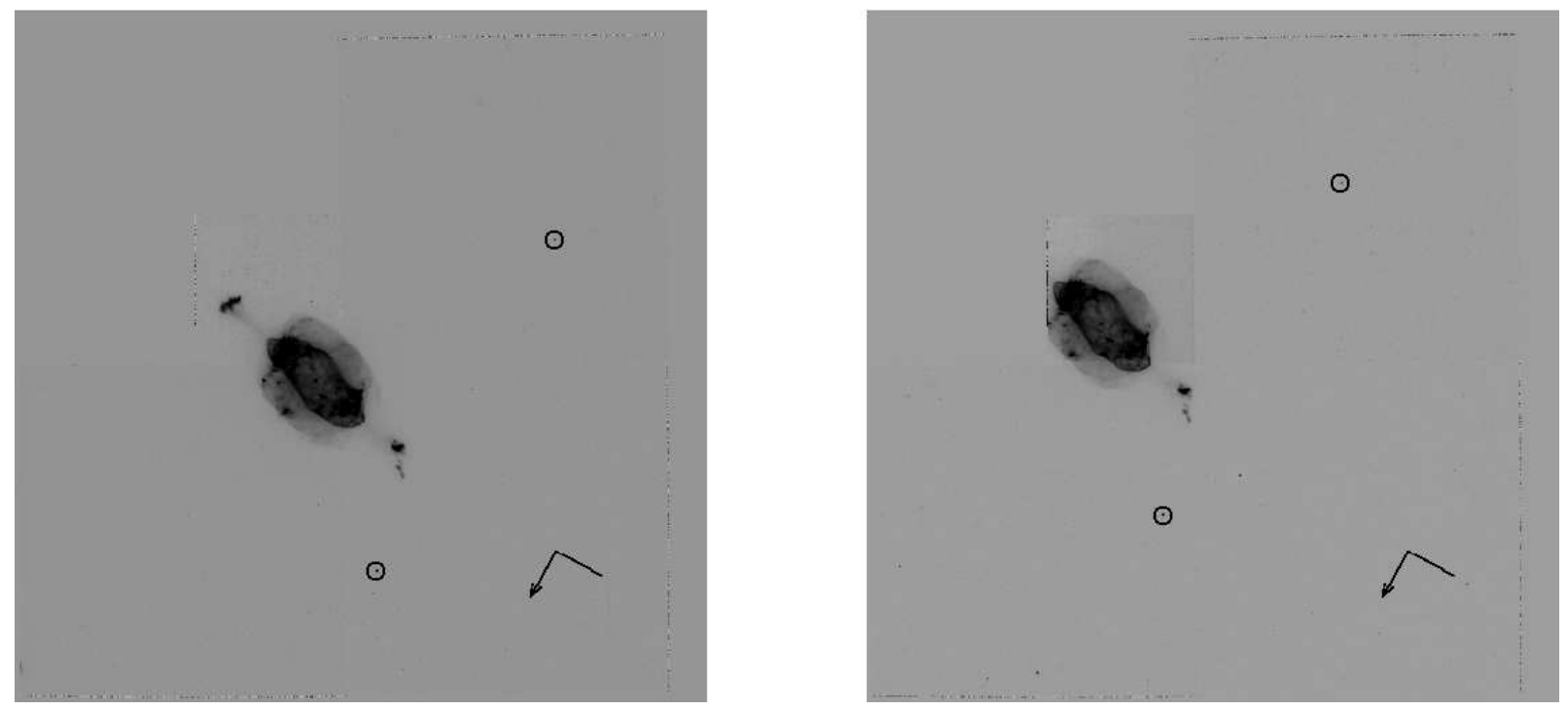

Fig. 1.- Average of the set of HST images taken in 1996 (left) and 2001 (right). North is down-left and east is down-right, and each image is 120 " by 120 ". There is a shift of 20 " in the east-west direction between them, which makes the western ansa disappear from the 2001 images. The two background stars marked with circles were used together with the CS as reference points for proper motion calculation.

\section{Results}

\subsection{Distance \& proper motion}

The distance to NGC 7009 is uncertain, but we computed the weighted average of the 14 available (Acker et al. 1992) values to be $d=0.86 \pm 0.34 \mathrm{kpc}$.

The measurement of the central star's position was made with the IRAF task imcentroid. For the measurement of the position of the ansa, we confined it inside a box of 5" square and computed the centroid, using the imcentroid task and measured their displacements with respect to the central star (CS) and two field stars (shown in Figure 1) in two ways: the displacements were measured in all the images, and an average displacement was obtained for each epoch; next, the displacements were computed from the averages of the images for each epoch. The centroid positions given in pixel values were converted to RA and DEC using the metric task, within the stsdas package. This task corrects for the known geometric distortions between different CCDs. The differences in the results are an order of magnitude smaller than the errors derived from centroid calculation and measurements between different chips. The various differential images are shown in Figure 2. 


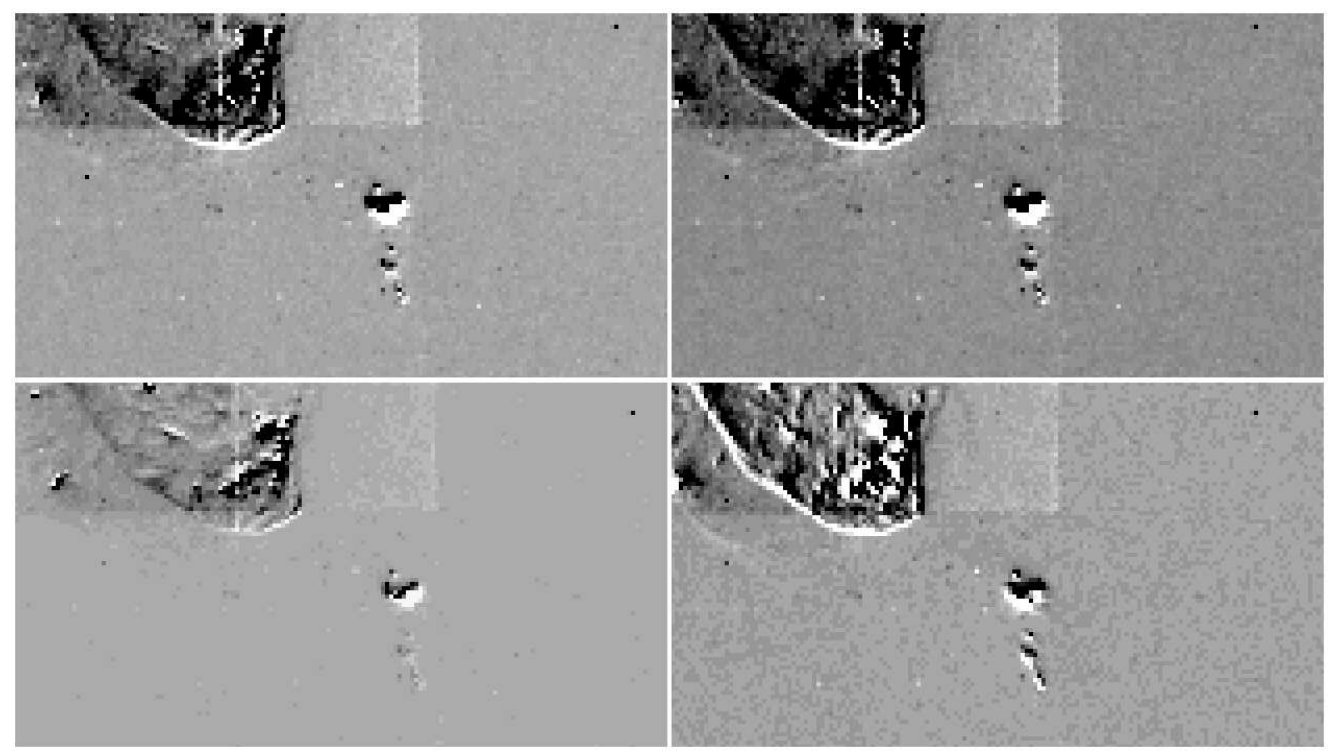

Fig. 2.- Difference between the combined 2001 and 1996 images, normalized by the average flux of the three reference points chosen: central star and the two field stars. The panels show differences wrt: central star (upper-right), field star 1 (lower-left), field star 2 (lower right) and the adopted least squares fit (upper-left). Each image is 43 " by 24 ". 
Table 1: The relative separation between central star and eastern ansa for each of the images.

1996 images

\begin{tabular}{lcl} 
Dataset & UT of exp. & Relative separation (") \\
\hline u32e0308t & $20: 48: 16$ & $24.85 \pm 0.04$ \\
u32e0309t & $20: 56: 16$ & $24.84 \pm 0.04$ \\
u32e030at & $21: 04: 16$ & $24.86 \pm 0.04$ \\
Average & $20: 56: 16$ & $24.85 \pm 0.06$ \\
\hline
\end{tabular}

2001 images

\begin{tabular}{lcl} 
Dataset & UT of exp. & Relative separation (") \\
\hline u5hc6002r & $19: 05: 14$ & $25.01 \pm 0.04$ \\
u5hc6003r & $19: 13: 14$ & $25.00 \pm 0.04$ \\
u5hc6004r & $19: 24: 14$ & $25.00 \pm 0.04$ \\
u5hc6005r & $19: 31: 14$ & $25.02 \pm 0.04$ \\
Average & $19: 18: 29$ & $25.01 \pm 0.05$ \\
\hline
\end{tabular}

The displacements between the CS and the ansae are: $24.85 \pm 0.06^{\prime \prime} \& 25.01 \pm 0.05^{\prime \prime}(\mathrm{E}$; $1996 \& 2001)$ and $26.77 \pm 0.06^{\prime \prime}(\mathrm{W} ; 1996$ only). The detailed measurements with their respective errors are shown in Table 1. Note that the adjusted standard deviations for 1996 and 2001 results are 0.01 and $9.6 .10^{-3}$ respectively.

Even though this is a good determination for the angular displacement of the eastern ansa, it is also possible that the central star is moving. Therefore, it is important to have other reference points. The circles in Figure 1 indicate the position of the two field stars present on the images that could be measured. The angular displacements of the ansae in each frame are listed in Table 2. These measurement are all equal within the errors.

The value we adopted for the displacement was the least squares shift of all measurements: $0^{\prime \prime} .14 \pm 0^{\prime \prime} .04$. The time between images is $158883733 \mathrm{~s}$ or $\approx 5$ yrs. computed from the start time of the exposures, yielding a proper motion of the $\mathrm{E}$ ansa of $0^{\prime \prime} .028 \pm 0^{\prime \prime} .008$ $\mathrm{yr}^{-1}$, and the tangential velocity is $\mathrm{V}_{\exp }=112 \pm 32 \mathrm{~km} \cdot \mathrm{s}^{-1}$ for $\mathrm{d}=860 \mathrm{pc}$.

We note that the rim of the inner nebulae also shows differential motion in more or less the same direction as that of the ansae between the 1996 and 2001 images. We do not attempt to compute the proper motion as this rim is likely to be a moving ionization front 
Table 2: Angular displacements of the east ansa as measured from the combined 1996 and 2001 images using the reference points specified (Upper star and Lower star refer to Figure 1).

\begin{tabular}{lc}
\hline Reference Point & Angular displacement (") \\
\hline \hline Central star & $0.17 \pm 0.10$ \\
Upper star & $0.16 \pm 0.10$ \\
Lower star & $0.10 \pm 0.02$ \\
Average shift & $0.13 \pm 0.04$ \\
Least squares shift & $0.14 \pm 0.04$ \\
\hline
\end{tabular}

with a very different velocity than that of the bulk motion of the gas (weak R-front, Mellema (2003)). This is unlikely to be the case for the ansae.

\subsection{Radial Velocities}

We computed the mean observed radial velocities of the ansae from 10 emission lines fitted with a Voigt profile to be $-58.5 \pm 1.9 \mathrm{~km} . \mathrm{s}^{-1}$ (E) and $-47.9 \pm 1.6 \mathrm{~km} . \mathrm{s}^{-1}$ (W). When using only the $[\mathrm{NII}]$ and $\mathrm{H} \alpha$ lines (the HST images were taken in these lines) we obtain $-58.3 \pm 1.1 \mathrm{~km} . \mathrm{s}^{-1}(\mathrm{E})$ and $-47.7 \pm 0.8 \mathrm{~km} . \mathrm{s}^{-1}(\mathrm{~W})$. The radial velocity differences between the $\mathrm{E}$ and $\mathrm{W}$ ansae are: $\Delta_{H \alpha[N I I]}=10.6 \pm 1.4 \mathrm{~km} . \mathrm{s}^{-1}$ and $\Delta_{A l l}=10.6 \pm 2.5 \mathrm{~km} . \mathrm{s}^{-1}$. We also separately computed the mean values of the velocity for the forbidden and for the permitted lines. They were also the same to within the errors. The observed radial velocities for each detected emission line are listed in Table 3, and we also give the heliocentric and LSR velocities and their average values.

\section{3. $\quad$ Electron temperatures and densities}

The line intensities of [NII] and [SII] were measured by fitting Voigt profiles to derive the electron temperature and density in both ansae. The line intensities were corrected for reddening, assuming a recombination model $\mathrm{B}$ with $T=10^{4} \mathrm{~K}$ and $n_{e}=10^{4} \mathrm{~cm}^{-3}$, and an extinction coefficient $c_{\beta}=0.26$ (Lame \& Pogge 1996). The line intensity ratios together with electron temperatures and densities derived from them are listed in Table 4 . The latter results were obtained using the formulae from McCall (1984). The errors in the ratios were calculated from the $\mathrm{S} / \mathrm{N}$ in each line. 
Table 3: Radial velocities in $\mathrm{km} \cdot \mathrm{s}^{-1}$ for each emission line identified.

\begin{tabular}{lcccccc}
\hline & eastern ansa & \multicolumn{5}{c}{ western ansa } \\
\hline Line & $\mathrm{V}_{\text {obs }}$ & $\mathrm{V}_{\text {hel }}$ & $\mathrm{V}_{l s r}$ & $\mathrm{~V}_{\text {obs }}$ & $\mathrm{V}_{\text {hel }}$ & $\mathrm{V}_{l s r}$ \\
\hline$[\mathrm{NII}] 5755$ & -59.17 & -55.43 & -45.42 & -47.70 & -43.71 & -33.73 \\
$\mathrm{HeI} 5876$ & -56.42 & -52.68 & -42.67 & -48.51 & -44.56 & -34.54 \\
{$[\mathrm{OI}] 6364$} & -62.23 & -58.49 & -48.48 & -50.44 & -46.49 & -36.47 \\
{$[\mathrm{OI}] 6300$} & -61.19 & -57.45 & -47.44 & -49.05 & -45.10 & -35.08 \\
{$[\mathrm{NII}] 6548$} & -59.33 & -55.59 & -45.58 & -48.34 & -44.39 & -34.37 \\
$\mathrm{H} \alpha 6563$ & -56.69 & -52.95 & -42.94 & -48.46 & -44.51 & -34.49 \\
{$[\mathrm{NII}] 6583$} & -57.87 & -54.13 & -44.12 & -46.48 & -42.53 & -32.51 \\
{$[\mathrm{SII}] 6716$} & -57.68 & -53.94 & -43.93 & -45.34 & -41.39 & -31.37 \\
{$[\mathrm{SII}] 6731$} & -56.83 & -53.09 & -43.08 & -45.46 & -41.51 & -31.49 \\
{$[\mathrm{AIII}] 7134$} & -57.60 & -53.86 & -43.85 & -48.97 & -45.02 & -35.00 \\
Averages & $-58.5 \pm 1.9$ & $-54.76 \pm 1.9$ & $-44.75 \pm 1.9$ & $-47.9 \pm 1.6$ & $-43.92 \pm 1.6$ & $-33.91 \pm 1.6$ \\
\hline
\end{tabular}

Table 4: Derived line properties.

Eastern ansa

\begin{tabular}{lccl} 
Ratio & Observed & Corrected & Result \\
\hline$[\mathrm{NII}](6548+6583) / 5755$ & $111 \pm 15$ & $103 \pm 15$ & $T_{e}=9000 \pm 400 \mathrm{~K}$ \\
{$[\mathrm{SII}] 6716 / 6730$} & $0.67 \pm 0.04$ & $0.67 \pm 0.04$ & $n_{e}=2600 \pm 500 \mathrm{~cm}^{-3}$ \\
\hline
\end{tabular}

Western Ansa

\begin{tabular}{lccl} 
Ratio & Observed & Corrected & Result \\
\hline$[\mathrm{NII}](6548+6583) / 5755$ & $120 \pm 12$ & $110 \pm 12$ & $T_{e}=8900 \pm 400 \mathrm{~K}$ \\
{$[\mathrm{SII}] 6716 / 6730$} & $0.64 \pm 0.04$ & $0.64 \pm 0.04$ & $n_{e}=1900 \pm 300 \mathrm{~cm}^{-3}$ \\
\hline
\end{tabular}

\section{Discussion}

Our radial velocities agree with those of Reay \& Atherton (1985), who determined $\pm 6.2 \mathrm{~km} \cdot \mathrm{s}^{-1}$ using the [OI]6300 line. We get $\pm 6.1 \mathrm{~km} \cdot \mathrm{s}^{-1}$ using the same line. 
Liller (1965) computes 16 mas.yr $^{-1}$ for the angular expansion of the ansae; we obtain $28 \pm 8$ mas. $\mathrm{yr}^{-1}$. This difference is probably due to the low resolution of the photographic plates, as Liller comments: "....the plate scale is such that photographic grain often competes with seeing as the limiting factor in image definition".

If the ansae were ejected at the same time from the CS and with equal \& opposite velocities, we can determine the PM of the CS (relative to the point of ejection, assumed fixed in space) and the age of the ansae from our data. We find that the CS has moved $0.96 \pm 0.04$ " since the ejection of the ansae, $920 \pm 260$ yrs ago. This gives a proper motion for the CS of $1 \pm 0.5$ mas. $\mathrm{yr}^{-1}$ or 5 mas between our two epochs, negligible cf. the motion of the ansae.

In the gas of NGC7009, the sound speed is about $10 \mathrm{~km} \cdot \mathrm{s}^{-1}$, implying that the ansae move at supersonic speeds.

The $\mathrm{T}_{e}$ and $\mathrm{n}_{e}$ we obtain are similar to those found by others: Balick et al. (1994) quote a value of $\mathrm{T}_{e}=8100 \mathrm{~K} \& \mathrm{n}_{e}=1000 \mathrm{~cm}^{-3}$; Bohigas, López \& Aguilar (1994) quote a value of $\mathrm{T}_{e}=9800 \mathrm{~K} \& \mathrm{n}_{e}=2300 \mathrm{~cm}^{-3}$, both for the $\mathrm{W}$ ansa.

\section{Conclusions}

We conclude that the ansae in NGC7009 -which is at a distance of $860 \pm 340$ pc- move outward at the supersonic velocity of $\pm 112 \mathrm{~km} . \mathrm{s}^{-1}$ near the plane of the sky ( $2.6 \pm 1$ degrees), have a dynamic age of $920 \pm 260$ yrs, a proper motion of $28 \pm 8 \mathrm{mas}_{\mathrm{yr}}{ }^{-1}$, and that the CS likely moves at $1 \pm 0.5$ mas. $\mathrm{yr}^{-1}$.

\section{REFERENCES}

Aller, L. H. 1941, ApJ, 93, 236

Acker, A. et al. 1992, Strasbourg-ESO Catalogue of Galactic PNe (Garching:ESO)

Balick, B. 1987, AJ, 94, 671

Balick, B., Preston, H. \& Icke, V. 1987, AJ, 94, 1641

Balick, B. et al. 1994, ApJ, 424, 800

Balick, B. et al. 1998 AJ, 116, 360 
Bohigas, J. López, J. \& Aguilar, L. 1994, A\&A, 291, 595

Corradi, R.L.M. \& Schwarz, H.E. 1995 A\&A, 293,871

Hamuy, M. et al. 1992 PASP, 104, 533

Iben, I. \& Renzini, A. 1983 ARAA, 21, 271

Kastner, J.H., Soker, N. \& Rappaport, S. 2000, ASP Conf.Ser. 199, "Asymmetrical PNe II: From Origins to Microstructures"

Kwok, S., Purton, C.R. \& Fitzgerald, P.M. 1978 ApJ, 219, L125

Lame, N. \& Pogge, R. 1996, AJ, 111, 2320

Liller, W. 1965, PASP, 77, 25

López, J.A. 1997 IAUS 180, 197

McCall, M. 1984, MNRAS, 208, 253.

Mellema, G. 2003 private communication.

Palen, S. et al. 2002 AJ, 123, 2666

Reay, N. K. and Atherton, P. D. 1985, MNRAS, 215, 233

Sahai, R., Trauger, J.T. 1998 AJ, 116, 1357

Schwarz, H.E., Corradi, R.L.M. \& Melnick, J. 1992, A\&AS, 96, 23

Schwarz, H.E. 1993, in: ESO Workshop Proc.46, "Mass Loss on the AGB and Beyond", 223

This preprint was prepared with the AAS LATEX macros v5.0. 